\title{
Recognition without awareness: An elusive phenomenon
}

\author{
Annette Jeneson, ${ }^{1}$ C. Brock Kirwan, ${ }^{4}$ and Larry R. Squire ${ }^{1,2,3,5,6}$ \\ ${ }^{7}$ Department of Psychology, University of California, San Diego, California 92093, USA; ${ }^{2}$ Department of Psychiatry, University of \\ California, San Diego, California 92093, USA; ${ }^{3}$ Department of Neurosciences, University of California, San Diego, California 92093, \\ USA; ${ }^{4}$ Department of Psychology and Neuroscience Center, Brigham Young University, Provo, Utah 84602, USA; ${ }^{5}$ Veterans Affairs \\ Healthcare System, San Diego, California 92161, USA
}

\begin{abstract}
Two recent studies described conditions under which recognition memory performance appeared to be driven by nondeclarative memory. Specifically, participants successfully discriminated old images from highly similar new images even when no conscious memory for the images could be retrieved. Paradoxically, recognition performance was better when images were studied with divided attention than when images were studied with full attention. Furthermore, recognition performance was better when decisions were rated as guesses than when decisions were associated with low or high confidence. In three experiments, we adopted the paradigm used in the earlier studies in an attempt to repeat this intriguing work. Our attempts were unsuccessful. In all experiments, recognition was better when images were studied with full attention than when images were studied with divided attention. Recognition was also better when participants indicated high or low confidence in their decision than when they indicated that their decision was a guess. Thus, our results conformed to what typically has been reported in studies of recognition memory, and we were unable to demonstrate recognition without awareness. We encourage others to explore this paradigm, and to try to identify conditions under which the phenomenon might be demonstrated.
\end{abstract}

Declarative memory refers to the capacity to recollect facts and events, and can be contrasted with a collection of nondeclarative memory abilities, including skills, habits, and the phenomenon of priming, which are expressed through performance rather than recollection (Squire et al. 2004). Declarative memory depends on the integrity of medial temporal lobe structures, while the various forms of nondeclarative memory depend on other brain systems (Schacter and Tulving 1994; Eichenbaum and Cohen 2001; Squire 2004). The best-studied example of declarative memory is recognition memory-the ability to judge items as having been encountered previously. Successful recognition is ordinarily accompanied by a conscious experience of familiarity, and sometimes by conscious memory of the prior encounter itself (Gabrieli 1998).

One interesting idea that has been explored in some detail is that recognition memory decisions based on familiarity might also benefit from priming. Priming refers to an improved ability to produce or identify an item on the basis of a recent encounter with the same item or a related item, but without a requirement that there be conscious knowledge of the prior encounter (Tulving and Schacter 1990; Schacter and Buckner 1998). In early studies, it was suggested that previously encountered items might be processed more fluently (e.g., with greater speed and with more ease), and that improved fluency might influence familiarity judgments. Specifically, items perceived with greater fluency might tend to be identified as familiar (Mandler 1980; Jacoby and Dallas 1981; Johnston et al. 1991).

This idea encountered difficulty when it was found that severely amnesic patients can perform at chance on conventional recognition tests despite exhibiting intact perceptual priming

\footnotetext{
${ }^{6}$ Corresponding author.
}

E-mail Isquire@ucsd.edu; fax (858) 552-7457.

Article is online at http://www.learnmem.org/cgi/doi/10.1101//m.1815010.
(Hamann and Squire 1997; Stark and Squire 2000). If fluency facilitates recognition, severely amnesic patients who exhibit intact perceptual priming should perform better than chance on recognition memory tests. Thus, it has seemed that the perceptual fluency that mediates priming does not also support familiaritybased recognition judgments, at least not to a measurable degree. Indeed, the contribution of perceptual fluency appears to be too weak to drive recognition performance noticeably above chance (Conroy et al. 2005).

Nonetheless, it remains possible that conditions might be found under which recognition decisions can benefit from perceptual fluency, and in this way be linked to nondeclarative memory. Two recent studies (Voss et al. 2008; Voss and Paller 2009) described conditions under which recognition memory appeared to be significantly driven by nondeclarative memory. Participants studied difficult-to-verbalize images (Fig. 1) with either full attention or divided attention. At test, each image was paired with a highly similar new image, and participants made a speeded forced-choice decision. The striking finding was that, under these conditions, accurate recognition memory performance occurred, but without the awareness that ordinarily accompanies successful recognition. Specifically (and paradoxically), performance was better under divided-attention conditions (which ordinarily degrade memory performance) than under full-attention conditions (Fig. 2A). Furthermore, in one study (Voss et al. 2008), recognition was better when participants reported that they were guessing than when they reported conscious memory of the images (combined high- and low-confidence trials) (Fig. 2B). Notably, this phenomenon occurred only when the test was given in a forced-choice format, and not in a yes/no format. The other study (Voss and Paller 2009) reported a similar advantage for guessing in the divided-attention condition. These two reports appear to demonstrate recognition without awareness and a significant contribution of nondeclarative memory to recognition performance. 

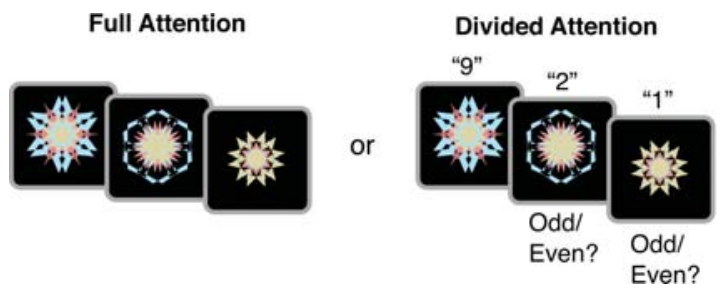

Figure 1. In the full-attention condition, participants studied 14 images for 2 sec each (1.5-sec intertrial interval). Alternatively, in the divided-attention condition, participants studied the images while deciding whether a digit heard during the previous trial was odd or even. The forced-choice recognition test probed memory for the middle 10 images presented in the study sequence. Each studied item was presented together with a highly similar new item, and participants selected the old item by responding "left" or "right." After each response, participants indicated how confident they were in their recognition decision ( $G$, guess; $L$, low confidence; $H$, high confidence).

These findings challenge the conventional view that recognition memory is more effective when full attention is given to a task than when attention is divided (Anderson 1980), that recognition memory is associated with a conscious experience of familiarity (Gabrieli 1998), and that recognition memory accuracy is positively correlated with ratings of confidence (Reed et al. 1997; Mickes et al. 2007). Because the reported findings are exceptional, we explored the phenomenon further in three separate experiments in an attempt to replicate it and identify its boundary conditions. We adopted the same paradigm as was used in the original study (Voss et al. 2008).

\section{Results}

To facilitate comparison with the earlier work, our figures show $d^{\prime}$ (discriminability) values to show overall performance under full and divided attention (A in each figure), and percent correct scores to show performance in relation to confidence ratings (B in each figure).

\section{Experiment 1}

Accuracy $\left(d^{\prime}\right)$ was numerically higher in the full-attention condition than in the divided-attention condition (Fig. 3A), but the difference was not significant $(P>0.10)$, probably due to the small sample size (see Experiment 2, below). Performance was better than chance (i.e., above zero) in both conditions $\left[t_{(6)}>2.8\right.$, $P$ s $<0.05]$. The results were the same when accuracy was measured as proportion correct ( $66.2 \%$ vs. $62.5 \%)$.

To analyze accuracy as a function of confidence rating, lowconfidence and high-confidence responses were combined in
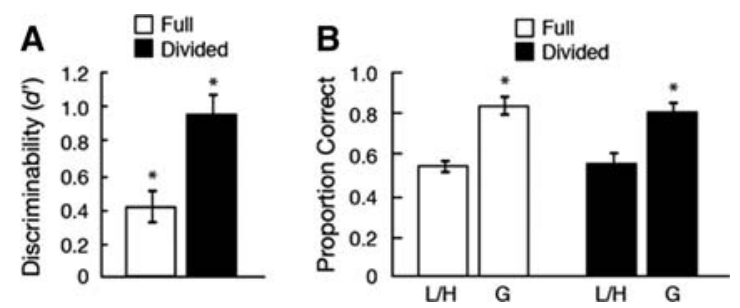

Figure 2. Data from Experiment 2 in Voss et al. (2008), estimated from their Figure 2. $(A)$ When recognition was probed using a forced-choice format, performance was more accurate in the divided-attention condition than in the full-attention condition. (B) In both conditions, forced-choice recognition was more accurate in trials where participants indicated that their recognition decision was a guess $(G)$ than in trials where participants indicated low or high confidence $(\mathrm{L} / \mathrm{H})$ in their decision. Asterisks indicate performance significantly above chance $(P<$ 0.05). Error bars indicate SEM.
Forced Choice Test

\section{Confidence}

G L H

Left/Right?

\section{.}



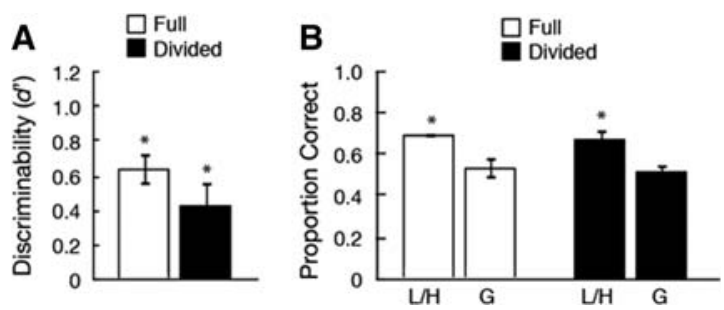

Figure 3. Experiment $1(N=7)$. (A) Performance was more accurate in the full-attention condition than in the divided-attention condition. $(B)$ In both conditions, forced-choice recognition was more accurate in trials where participants indicated low or high confidence $(\mathrm{L} / \mathrm{H})$ in their decision than in trials where participants indicated that their decision was a guess $(G)$. Asterisks indicate performance significantly above chance $(P<0.05)$. Error bars indicate SEM.

condition than in the divided-attention condition (Fig. 4B). Thus, we found no evidence for unaware recognition memory.

The mean reaction time for the recognition decisions was $318 \pm 17 \mathrm{msec}$ (this excludes 2.4 trials/participant, which were discarded from the data analyses because response times were longer than $1.5 \mathrm{sec}$ ). Participants were $93.3 \% \pm 1.8 \%$ accurate in performing the distracting digit task.

We next examined data from the seven participants (out of 22) who provided the highest proportion of guesses in the divided-attention condition (43.0\% guesses). There was no evidence of recognition without awareness in this group of participants, and their scores were similar to the scores presented in Figure 4. Specifically, accuracy was higher in the full-attention condition than in the divided-attention condition, and accuracy was higher when these participants indicated low or high confidence in their decision than when they indicated that their decision was a guess (full-attention condition: $79.2 \%$ vs. $53.3 \%$ correct; divided-attention condition: $74.7 \%$ vs. $53.2 \%$ correct).

Because the test material was identical to the material from the earlier study (Voss et al. 2008) for only six of the 16 study-test blocks (we created the material for the other 10 blocks), we also analyzed the data separately for the two kinds of material. In the two attention conditions, overall accuracy was the same for the tests based on the two kinds of material (differences within $2.2 \%)$. Critically, the incidence of guess trials was also the same (within 3.1\%), and the accuracy scores for guess trials and for low-/high-confidence trials were about the same (within $8 \%$ for each attention condition).

Lastly, because 80 forced-choice trials were administered in the current experiment and only 10 forced-choice trials were administered in the previous study (Voss et al. 2008), we scored

Table 1. Percent of decisions associated with guesses vs. low/high confidence

\begin{tabular}{|c|c|c|c|c|}
\hline & \multicolumn{2}{|c|}{ Full Attention } & \multicolumn{2}{|c|}{ Divided Attention } \\
\hline & Guess & Low/High & Guess & Low/High \\
\hline Voss et al. (2008) & 16 & 84 & 58 & 42 \\
\hline $\begin{array}{l}\text { Voss and Paller } \\
\text { (2009) }\end{array}$ & 23 & 77 & 45 & 55 \\
\hline $\begin{array}{l}\text { Experiment } 1 \\
(N=7)\end{array}$ & $20.4(3.6)$ & $79.6(3.6)$ & $27.5(4.7)$ & $72.5(4.7)$ \\
\hline $\begin{array}{l}\text { Experiment } 2 \\
\quad(N=22)\end{array}$ & $16.8(2.4)$ & $83.2(2.4)$ & $24.2(3.2)$ & $75.8(3.2)$ \\
\hline $\begin{array}{l}\text { Experiment } 3 \\
\quad(N=24)\end{array}$ & $15.5(3.6)$ & $84.5(3.6)$ & $24.1(4.2)$ & $75.9(4.2)$ \\
\hline
\end{tabular}

Parentheses show SEM. Values from the earlier studies (Voss et al. 2008; Voss and Paller 2009) are estimated from their figures. each participant's performance for only the first 10 trials of each attention condition. The results were the same as when data from all trials were scored (i.e., better recognition with full attention $\left[d^{\prime}=1.03\right]$ than with divided attention $\left[d^{\prime}=0.49\right]$, and better recognition when participants indicated high or low confidence in their decision than when they indicated their decision was a guess [full attention: $75.3 \%$ vs. $56.5 \%$ correct; divided attention: $68.4 \%$ vs. $58.3 \%$ correct, respectively]).

\section{Experiment 3}

Experiment 3 was designed to match the earlier study (Voss et al. 2008) as closely as possible in every respect (only two test blocks in each attention condition and the addition of a yes/no test). Because the yes/no data are not relevant to the phenomenon under study, only the forced-choice data are reported here.

The findings were the same as in the first two experiments (Fig. 5). Accuracy was higher in the full-attention condition than in the divided-attention condition $\left[t_{(23)}=2.6, P<0.05\right]$ (Fig. 5A), but better than chance in both conditions (Ps $<0.05)$. The results were the same when accuracy was measured as proportion correct (68.0\% vs. $58.3 \%)$.

An analysis of variance (full vs. divided attention and low/ high confidence vs. guess) revealed a main effect of condition $\left[F_{(1,12)}=5.9, P<0.05\right]$, a main effect of confidence $\left[F_{(1,12)}=6.0\right.$ $P=0.05]$, and no condition $\times$ confidence interaction. The effect of condition indicates that accuracy was higher overall in the fullattention condition than in the divided-attention condition (Fig. 5A). The effect of confidence indicates that accuracy was higher overall when participants indicated low or high confidence in their decision than when they indicated that their decision was a guess (full-attention condition: $71.4 \%$ vs. $55.0 \%$ correct; divided-attention condition: $61.9 \%$ vs. $42.7 \%$ correct, $P=0.06$ and 0.08) (Fig. 5B).

Mean reaction time for recognition responses was $552 \pm 32$ msec (this excludes 1.6 trials/participant, which were excluded from all analyses because response times were longer than 1.5 sec). Participants were $92.8 \% \pm 2.3 \%$ accurate in performing the distracting digit task.

Because participants completed only 10 test trials for each condition, some participants did not rate any decisions as a guess. We therefore examined the data separately for those 13 of 24 participants who had guess decisions and low-/high-confidence decisions in both attention conditions. The results obtained for all participants and for this subset of 13 participants were about the same (all differences within 5.2\%).

\section{Discussion}

Two recent studies (Voss et al. 2008; Voss and Paller 2009) described conditions under which recognition appeared to be significantly driven by nondeclarative memory. Under these conditions (difficult-to-verbalize stimuli, highly similar targetfoil pairs, speeded responding), recognition memory performance was better when images were studied with divided attention than when images were studied with full attention. Furthermore, recognition was better when participants reported they were guessing than when they reported a conscious experience of familiarity with the images (combined high- and low-confidence trials [Voss et al. 2008] or combined Remember and Know trials [Voss and Paller 2009]). These findings are exceptional in that recognition is ordinarily better when full attention is given to a task than when attention is divided, recognition is ordinarily accompanied by a conscious experience of familiarity, and recognition memory accuracy is ordinarily positively correlated with ratings 

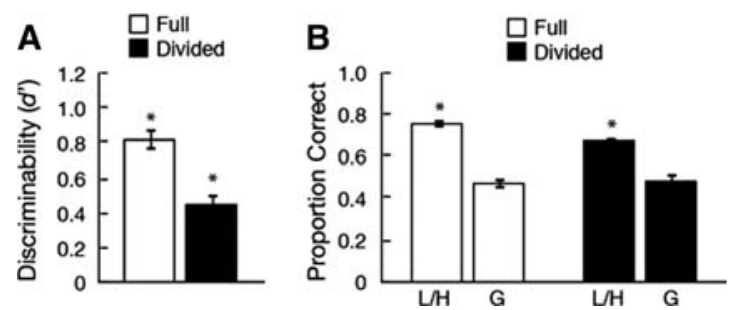

Figure 4. Experiment $2(N=22)$. The results were the same as in Experiment 1. (A) Performance was more accurate in the full-attention condition than in the divided-attention condition. (B) In both conditions, forced-choice recognition was more accurate in trials where participants indicated low or high confidence $(\mathrm{L} / \mathrm{H})$ in their decision than in trials where participants indicated that their decision was a guess (G). Asterisks indicate performance significantly above chance $(P<0.05)$. Error bars indicate SEM.

of confidence (Anderson 1980; Reed et al. 1997; Gabrieli 1998; Mickes et al. 2007).

In three experiments, we adopted the paradigm used in the earlier studies (Voss et al. 2008; Voss and Paller 2009) in an attempt to repeat the earlier work. The results were the same in all three experiments. Recognition was better when images were studied with full attention than when images were studied with divided attention. Recognition was also better when participants indicated low or high confidence in their decision than when they indicated that their decision was a guess. Furthermore, when participants indicated that their decision was a guess, performance was at chance levels. Thus, our results conformed to what is ordinarily found in studies of recognition memory, and we were unable to replicate the earlier studies.

What might explain the difference in results between our study and the earlier studies? We first considered whether participants in our experiments performed under sufficient time pressure. In one of the earlier studies, responses labeled as guesses were accurate only when participants were instructed to respond rapidly (Experiment 3 in Voss et al. [2008]). Perhaps slow responding could explain our failure to find good recognition performance in association with guesses. However, the mean response times in our experiments were similar to the mean reported by Voss and Paller (2009) (462 $\pm 112 \mathrm{msec}$ vs. $348 \pm 36 \mathrm{msec}$, $318 \pm 17 \mathrm{msec}$, and $552 \pm 32 \mathrm{msec}$ for our Experiments 1, 2, and 3 , respectively; response times were not reported in Voss et al. [2008]). Thus, it does not appear that slow responding can explain our results.

It also does not appear that the nature of the stimulus materials could explain the difference in results. First, in Experiment 3, the materials were identical to those used previously (Voss et al.
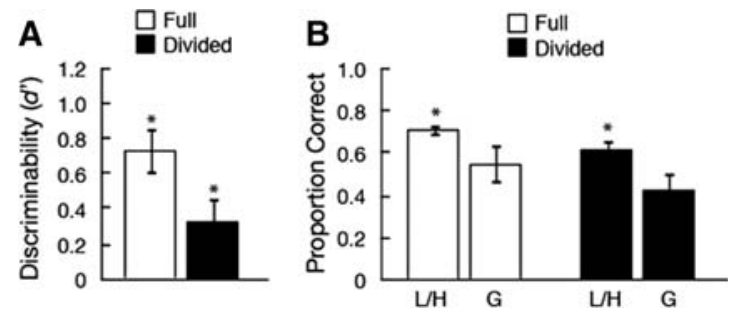

Figure 5. Experiment $3(N=24)$. The results were the same as in Experiments 1 and 2. (A) Performance was more accurate in the fullattention condition than in the divided-attention condition. $(B)$ In both conditions, forced-choice recognition was more accurate in trials where participants indicated low or high confidence $(\mathrm{L} / \mathrm{H})$ in their decision than in trials where participants indicated that their decision was a guess $(G)$. Asterisks indicate performance significantly above chance $(P<0.05)$. Error bars indicate SEM.
2008). Second, Experiment 2 did not replicate the earlier work, even when our data were scored separately for the 60 test trials (out of 160 trials) that used the same stimulus materials as were used originally.

In one of the earlier studies (Voss et al. 2008), participants completed only 10 forced-choice trials in each attention condition. In contrast, in our first two experiments, participants completed 80 forced-choice trials in each attention condition. Accordingly, we asked whether the greater number of trials we administered could account for the different results. This possibility seems unlikely. First, although one of the earlier studies did administer only 10 trials (Voss et al. 2008), the other study administered 56 trials (Voss and Paller 2009). Second, in our Experiment 2 we did not replicate the earlier work even when we scored only the first 10 trials of each attention condition. Third, in Experiment 3, participants completed only 10 trials, just as in one of the earlier studies (Voss et al. 2008). Thus, it does not appear that differences in the number of trials can explain the different results.

Although we did not find evidence of recognition without awareness in the average performance of any group, perhaps particular individuals demonstrated the phenomenon. Accordingly, we looked for individuals who exhibited better performance in the divided-attention condition than in the full-attention condition, as well as better performance for guess trials than for combined high- and low-confidence trials. Of the 53 participants tested, only two participants (both from Experiment 3) demonstrated both effects. However, for one of these individuals, performance was only slightly higher in the divided-attention condition $(67 \%$ correct) than in the full-attention condition (60\% correct). Furthermore, in the full-attention condition, this individual's performance was at chance for guesses. For the other individual, the score for guesses was based on only one trial. (No responses were labeled a guess in the full-attention condition.)

One potentially relevant difference between our study and the earlier studies is that participants in the divided-attention condition of our experiments were less likely to rate their decisions as a guess $(27.5 \%, 24.2 \%$, and $24.1 \%$ guesses in our Experiments $1-3$ vs. 58\% and 45\% guesses in Voss et al. [2008] and Voss and Paller [2009], respectively) (Table 1). Perhaps nondeclarative memory drives recognition performance only when the proportion of guesses is relatively high. In Experiment 2, we found no support for this idea in the case of the seven participants who provided the largest proportion of guesses in the divided-attention condition. We also explored the same idea across all three experiments (17 participants out of 53 who provided the highest proportion of guesses in the divided-attention condition: $45.6 \%$ guesses). There was no evidence of recognition without awareness in this larger group, and their scores were similar to the scores presented in Figures 3-5. That is, accuracy was higher in the fullattention condition than in the divided-attention condition, and accuracy was higher when these participants indicated low or high confidence in their decision than when they indicated that their decision was a guess (full-attention condition: $74.4 \%$ vs. $60.4 \%$ correct; divided-attention condition: $66.7 \%$ vs. $50.6 \%$ correct). Thus, it does not appear that the smaller proportion of guesses obtained in our study can explain our results.

A remaining possibility is that some subtle aspects of the instructions are crucial for eliciting recognition without awareness. In Experiments 2 and 3, we used the identical instructions from the earlier studies (Voss et al. 2008; Voss and Paller 2009), which directed participants in their use of confidence ratings and encouraged them to use their "gut feeling" (see Materials and Methods). Nevertheless, we cannot rule out the possibility that some differences remained in the way the instructions were delivered, or in the emphasis given to parts of the instructions, 
which were important for obtaining the phenomenon reported in the earlier studies.

In summary, our attempts to replicate recent reports that recognition can occur without awareness have been unsuccessful. Instead, our results are consistent with the conventional view that recognition is more effective when full attention is given to a task than when attention is divided, that successful recognition is accompanied by conscious memory, and that recognition memory accuracy is positively correlated with ratings of confidence (Anderson 1980; Reed et al. 1997; Gabrieli 1998; Mickes et al. 2007). Our findings suggest that the phenomenon we attempted to replicate is neither simple nor robust. Nevertheless, if there are conditions under which recognition without awareness can be observed, the findings would have important implications for theories of recognition memory. Accordingly, we urge others to attempt a replication of these intriguing reports (Voss et al. 2008; Voss and Paller 2009).

\section{Materials and Methods}

\section{Experiment 1}

\section{Participants}

Seven undergraduate students participated for course credit (six females, mean age $21.2 \pm 0.6$ yr of age).

\section{Materials and procedure}

This study was based on an earlier study of recognition memory (Experiment 2 in Voss et al. 2008). The stimuli consisted of 16 sets of 14 highly similar target/foil pairs of kaleidoscope images (i.e., each target was distinct, but each target had a highly similar foil) (Fig. 1). Six sets (84 targets, 84 foils) were the same as in the earlier study (Voss et al. 2008), and were generously provided by the investigators. An additional 10 sets, plus two sets used in practice blocks, were constructed using their methods.

Participants completed 16 study-test blocks (for each block, study 14 targets, test with 14 target/foil pairs). Images were studied either under a full-attention condition (eight blocks) or under a divided-attention condition (eight blocks). In the fullattention condition, participants studied 14 kaleidoscope images for $2 \mathrm{sec}$ each with an intertrial interval of $1.5 \mathrm{sec}$. The dividedattention condition was the same except that, while studying each image, participants decided whether a digit heard on the previous trial was odd or even (Fig. 1). Full-attention and dividedattention blocks were presented in random order, with the constraint that no condition was presented more than three times in succession. The order of presentation of stimulus sets was also randomized, as was the selection of which image of each target/ foil pair served as the target on each trial.

Recognition memory was tested after a 45-sec conversationfilled delay, using a forced-choice format. To reduce primacy and recency effects, only the 10 images that had been presented in the middle of the 14-image study sequence were presented at test. On each test trial, one of these targets was selected randomly and presented for 2 sec together with its highly similar foil. As soon as the two images had disappeared from the screen, participants were asked to identify the target as quickly as possible (as in Voss et al. 2008) (Fig. 1). Immediately after each recognition decision, participants were asked to report whether they had high confidence in their decision or low confidence, or whether they were simply guessing. After the confidence rating was given, there was a 1.5-sec delay before the next trial.

Prior to the first study-test block, participants completed two practice study-test blocks under divided attention. The following instructions were given regarding the confidence ratings:

Press " $\mathrm{H}$ " if you had high confidence in your memory response. For example, you would press " $\mathrm{H}$ " if you made the response based on memories of specific details of the image from the study phase.
Press "L" if you had low confidence in your memory response. Use "L" when your response was supported by only a weak feeling of familiarity, without being able to remember details of the image from the study phase.

Finally, press "G" if you were just randomly guessing because you had to make a response, as if you were flipping a coin to decide. So use "G" when you can't at all distinguish which of the two kaleidoscope images you saw during the study phase. The test is very hard, so don't feel bad if you feel like you're guessing a lot.

During the practice trials, participants received feedback on their response time for each decision in order to achieve response times shorter than $400 \mathrm{msec}$. After they had completed the practice blocks, participants were given additional instructions:

The test is very difficult, but we encourage you to continue to try as hard as possible even if you feel as though you are doing poorly on the test, because people often do better than they think on this task.

Participants did not receive response-time feedback during the 16 study-test blocks, but the importance of responding quickly (i.e., as quickly as during practice) was emphasized. Periodically, participants were reminded of the importance of responding quickly.

\section{Experiment 2}

\section{Participants}

Twenty-four undergraduate students participated for course credit (20 females, mean age $21.1 \pm 0.6 \mathrm{yr}$ of age).

\section{Materials and procedure}

These were the same as in Experiment 1, except that we used instructions kindly sent to us by the investigators of the earlier studies (Voss et al. 2008; Voss and Paller 2009):

Press $\mathrm{H}$ (for high confidence) if "you are confident in your decision because you remember a specific detail regarding the first time you saw the kaleidoscope during the study session. For instance, you might remember what you were thinking about when you saw it."

Press L (for low confidence) if "you are confident in your decision, but no details regarding the first time you saw the kaleidoscope come to mind. You selected the kaleidoscope because it felt in some way familiar to you."

Press G (for guess) if "you just guessed and did not pick the kaleidoscope based on a feeling of memory. You experienced absolutely no feeling of familiarity for the selected item, and were guessing because you were forced to select one kaleidoscope or the other."

Participants were given the following instructions after they had completed the practice blocks (as provided by the investigators of the earlier studies):

"The test is intended to be incredibly difficult, so don't worry if you feel like you are not doing well. Just try as best as you can and, if an answer does not come to you, just respond based on your gut feeling."

\section{Experiment 3}

\section{Participants}

Twenty-four undergraduate students participated for course credit (15 females, mean age $21.3 \pm 0.3 \mathrm{yr}$ of age). 


\section{Materials and procedure}

These were the same as in the earlier study (Experiment 2 in Voss et al. 2008). In this experiment, we attempted to replicate the earlier study in every respect. We used only four study-test blocks (14 study images in each block), and we included a yes/ no test, though this test was not relevant to the question of interest. For two study-test blocks, recognition was tested in a forced-choice format. For the other two study-test blocks, recognition was tested in a yes/no format. For the yes/no test, participants saw a list of 10 targets intermixed with 10 foils (each similar to one of the targets) and made an old/new decision for each image. Within each test format, one block was tested in the full-attention condition, and one block was tested in the divided-attention condition (the order of conditions was counterbalanced across participants). The four sets of material were used equally often in each condition.

Prior to the first study-test block, participants completed two practice blocks under the divided-attention condition, one of which was followed by a forced-choice test, and one by a yes/no test. As in the earlier studies (Voss et al. 2008; Voss and Paller 2009), no reaction-time feedback was provided during the practice trials (in contrast to our Experiments 1 and 2). Periodically, participants were reminded to make their responses quickly. Probably because no feedback was provided about reaction times, these times proved to be a little longer than in Experiments 1 and 2 (and similar to the times reported by Voss and Paller 2009).

\section{Acknowledgments}

We are grateful to Ken Paller and Joel Voss for constructive discussion, and for providing stimulus materials and instructions. We also thank Jennifer Frascino, Anna van der Horst, Ashley Knutson, and Flora Suh for research assistance, and Shlomi Sher, Christine Smith, and John Wixted for advice. This study was supported by the Medical Research Service of the Department of Veterans Affairs, the National Institute of Mental Health (MH24600), and the Metropolitan Life Foundation.

\section{References}

Anderson JR. 1980. Cognitive psychology and its implications. W.H. Freeman and Company, San Francisco, CA.
Conroy MA, Hopkins RO, Squire LR. 2005. On the contribution of perceptual fluency and priming to recognition memory. Cogn Affect Behav Neurosci 5: 14-20.

Eichenbaum H, Cohen NJ. 2001. From conditioning to conscious recollection: Memory systems of the brain. Oxford University Press, New York.

Gabrieli JD. 1998. Cognitive neuroscience of human memory. Annu Rev Psychol 49: 87-115.

Hamann SB, Squire LR. 1997. Intact priming for novel perceptual representations in amnesia. J Cogn Neurosci 9: 699-713.

Jacoby LL, Dallas M. 1981. On the relationship between autobiographical memory and perceptual learning. J Exp Psychol Gen 3: 306-340.

Johnston WA, Hawley KJ, Elliot MG. 1991. Contribution of perceptual fluency to recognition judgments. J Exp Psychol Learn Mem Cogn 17: 210-223.

Mandler G. 1980. Recognizing: The judgment of previous occurrence. Psychological Review 87: 252-271.

Mickes L, Wixted JT, Wais PE. 2007. A direct test of the unequal-variance signal detection model of recognition memory. Psychon Bull Rev 14: $858-865$.

Reed JM, Hamann SB, Stefanacci L, Squire LR. 1997. When amnesic patients perform well on recognition memory tests. Behav Neurosci 111: 1163-1170.

Schacter DL, Buckner RL. 1998. Priming and the brain. Neuron 20: 185-195

Schacter D, Tulving E. 1994. Memory systems 1994. MIT Press, Cambridge, MA.

Squire LR. 2004. Memory systems of the brain: A brief history and current perspective. Neurobiol Learn Mem 82: 171-177.

Squire LR, Stark CEL, Clark RE. 2004. The medial temporal lobe. Annu Rev Neurosci 27: 279-306.

Stark CEL, Squire LR. 2000. Recognition memory and familiarity judgments in severe amnesia: No evidence for a contribution of repetition priming. Behav Neurosci 114: $459-467$.

Tulving E, Schacter DL. 1990. Priming and human memory systems. Science 247: 301-306.

Voss JL, Paller KA. 2009. An electrophysiological signature of unconscious recognition memory. Nat Neurosci 12: 349-355.

Voss JL, Baym CL, Paller KA. 2008. Accurate forced-choice recognition without awareness of memory retrieval. Learn Mem 15: $454-459$.

Received March 22, 2010; accepted in revised form May 25, 2010. 


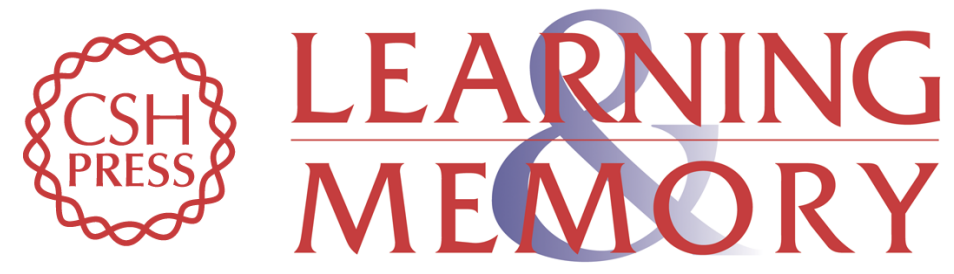

\section{Recognition without awareness: An elusive phenomenon}

Annette Jeneson, C. Brock Kirwan and Larry R. Squire

Learn. Mem. 2010, 17:

Access the most recent version at doi:10.1101//m.1815010

References This article cites 15 articles, 2 of which can be accessed free at: http://learnmem.cshlp.org/content/17/9/454.full.html\#ref-list-1

License

Email Alerting Receive free email alerts when new articles cite this article - sign up in the box at the Service top right corner of the article or click here. 